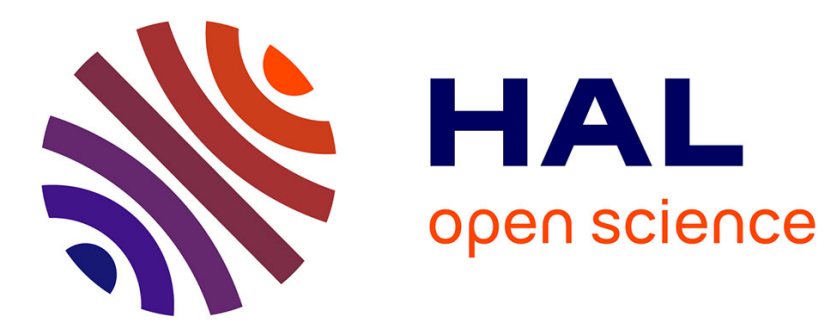

\title{
Nesting ocean models
}

Eric Blayo, Laurent Debreu

\section{To cite this version:}

Eric Blayo, Laurent Debreu. Nesting ocean models. Eric P. Chassignet; Jacques Verron. An Integrated View of Oceanography: Ocean Weather Forecasting in the 21st Century, Kluwer, 2006, 978-1-40204028-3. 10.1007/1-4020-4028-8_5. inria-00187576

\section{HAL Id: inria-00187576 https://hal.inria.fr/inria-00187576}

Submitted on 14 Nov 2007

HAL is a multi-disciplinary open access archive for the deposit and dissemination of scientific research documents, whether they are published or not. The documents may come from teaching and research institutions in France or abroad, or from public or private research centers.
L'archive ouverte pluridisciplinaire HAL, est destinée au dépôt et à la diffusion de documents scientifiques de niveau recherche, publiés ou non, émanant des établissements d'enseignement et de recherche français ou étrangers, des laboratoires publics ou privés. 


\title{
Chapter 6
}

\section{NESTING OCEAN MODELS}

\author{
Eric Blayo and Laurent Debreu \\ LMC-IMAG and INRIA Rhône-Alpes, Grenoble, France
}

\begin{abstract}
This note is focused on the problem of providing boundary conditions for regional ocean models. It is shown that usual methods generally do not address the correct problem, but more or less approaching ones. A tentative classification of these methods is proposed. Then their theoretical foundations are discussed, and recommendations are given.
\end{abstract}

Keywords: Open boundary conditions, regional models, nesting.

\section{Introduction}

The use of high resolution regional ocean models has become widespread in recent years, in particular due to the development of operational oceanography and coastal management systems. An important point, that has a strong influence on the quality of the results, is the way that a local model is forced at its open boundaries. Several methods, whose precise contents, theoretical justification, and practical performances are often somewhat difficult to compare precisely, are presently used in actual applications. In this context, the first aim of this note is to provide a tentative classification of these methods (section 1). Then we will discuss the one-way (section 2) and two-way (section 3) interactions, focusing on the theoretical foundations and practical use of the different approaches. Some final remarks on the available software tools and on the problem of data assimilation within nested models are given in sections 4 and 5 . 


\section{A classification of nesting problems}

\subsection{General framework}

We are interested in representing as accurately as possible the ocean in a local domain $\Omega_{l o c}$. The circulation is supposed to be described on a time period $[0, T]$ by a model which can be written symbolically

$$
L_{l o c} u_{l o c}=f_{l o c} \quad \operatorname{in} \Omega_{l o c} \times[0, T]
$$

with convenient initial conditions at $t=0 . L_{l o c}$ is a partial differential operator, $u_{l o c}$ is the state variable, and $f_{l o c}$ the model forcing. The conditions at the solid boundaries will never be mentioned in this note, since they do not interfere with our subject.

Since $\Omega_{l o c}$ is not closed, a portion of its boundary does not correspond to a solid wall, and has no physical reality. This artificial interface, also called open boundary (OB), is denoted $\Gamma$. The local solution $u_{l o c}$ is thus in interaction with the external ocean through $\Gamma$, and the difficulty consists in adequately representing this interaction in order to get a good approximation of $u_{l o c}$ in $\Omega_{l o c} \times[0, T]$.

We also assume that we have at our disposal a (probably less accurate) representation of the external ocean, either under the form of some data $u_{\text {ext }}$ or of an external model

$$
L_{e x t} u_{e x t}=f_{e x t} \quad \text { in } \Omega_{e x t} \times[0, T]
$$

where $\Omega_{e x t}$ is an external oceanic domain. Note that, in our notations, $\Omega_{l o c}$ and $\Omega_{e x t}$ do not overlap (Figure 1).

The best way to solve the local problem is then probably to use an inverse approach (e.g. Bennett, 2002), i.e. for example

Find $u_{l o c}$ that minimizes $\quad\left\|L_{l o c} u_{l o c}-f_{l o c}\right\|_{\Omega_{l o c} \times[0, T]}^{2}+\varepsilon\left\|u_{l o c}-u_{e x t}\right\|_{\Gamma \times[0, T]}^{2}$

where the norms are defined conveniently and take into account some statistical knowledge on the errors on $u_{e x t}$ and on the model (1), and where $\varepsilon$ is a weighting factor. One can also consider that the model is perfect, and minimize only $\left\|u_{l o c}-u_{e x t}\right\|_{\Gamma \times[0, T]}^{2}$, i.e. control the boundary values, under the constraint (1) (e.g. Taillandier et al., 2004).

However solving such an inverse problem is quite difficult and expensive. That is why ocean modellers usually use direct approaches. The goal is then to find $u_{l o c}$ satisfying (1) that connects adequately to $u_{e x t}$ through $\Gamma$. The mathematical formulation of this problem is generally not expressed clearly in actual applications. Since $\Gamma$ has no physical reality, the connection between $u_{e x t}$ and $u_{l o c}$ should be as smooth as possible, 
i.e. generally continuous and differentiable. Therefore a correct direct formulation of the problem can be the following :

Find $u_{l o c}$ that satisfies

$$
\begin{aligned}
& \left\{\begin{array}{c}
L_{l o c} u_{l o c}=f_{l o c} \quad \text { in } \Omega_{l o c} \times[0, T] \\
u_{l o c}=u_{e x t} \quad \text { and } \quad \frac{\partial u_{l o c}}{\partial n}=\frac{\partial u_{e x t}}{\partial n} \quad \text { on } \Gamma \times[0, T]
\end{array}\right. \\
& \text { in } \Omega_{e x t} \times[0, T]
\end{aligned}
$$

or equivalently :

$$
\begin{aligned}
& \text { Find } u_{l o c} \text { and } u_{e x t} \text { that satisfy } \\
& \left\{\begin{array}{c}
L_{l o c} u_{l o c}=f_{l o c} \text { in } \Omega_{l o c} \times[0, T] \quad \text { and } \quad L_{e x t} u_{e x t}=f_{e x t} \\
\text { in } \Omega_{e x t} \times[0, T] \\
\text { with } u_{l o c}=u_{e x t} \text { and } \quad \frac{\partial u_{l o c}}{\partial n}=\frac{\partial u_{e x t}}{\partial n} \quad \text { on } \Gamma \times[0, T]
\end{array}\right.
\end{aligned}
$$

where $n$ denotes the normal direction. However, in actual applications, the external model is not always available for online interaction. Moreover it is defined generally on $\Omega_{e x t} \cup \Omega_{l o c}$ (i.e. it fully overlaps the local domain), and it would be quite expensive to modify it in order to avoid this overlapping by implementing an open boundary on $\Gamma$. Therefore most applications generally do not address thecorrect problem (5) itself, but rather more or less approaching problems.

Remark : the operators $L_{e x t}$ and $L_{l o c}$ generally differ, both in their continuous form (e.g. subgrid scale paramaterizations) and in their discretized form (the local numerical model often has a higher resolution than the external model). Moreover the forcings $f_{\text {ext }}$ and $f_{l o c}$, and the discretized bathymetries defining $\Omega_{e x t}$ and $\Omega_{l o c}$ can be rather different. In that case the regularity conditions in (5) cannot be satisfied, and the connection between $u_{e x t}$ and $u_{l o c}$ is unsmooth, which is of course non-physical. That is why it is recommended to define the models and forcings in order to ensure as far as possible the smoothness of the transition between the two models. This can be done for instance into a transition zone defined in the vicinity of $\Gamma$.

\section{$2.2 \quad$ The different approaches}

The usual approaches can be classified as follows :

The open boundary problem. This is the usual case where the local model only is used. The problem writes

$$
\begin{cases}L_{l o c} u_{l o c}=f_{l o c} & \text { in } \Omega_{l o c} \times[0, T] \\ B u_{l o c}=g & \text { on } \Gamma \times[0, T]\end{cases}
$$




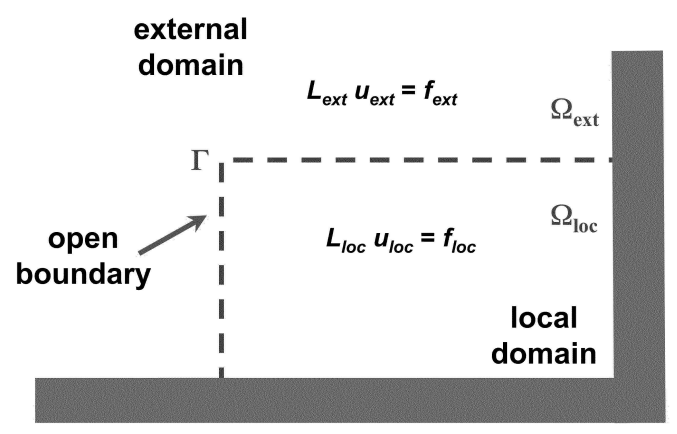

Figure 1. A schematic view of the nesting problem

where $B$ denotes an open boundary operator. The choice of $B$ and $g$ will be discussed in $\S 3$.

A particular case: one-way nesting. It is frequent that the solution $u_{\text {ext }}$ of an external model covering an area $\Omega_{\text {ext }} \cup \Omega_{l o c}$ larger than $\Omega_{l o c}$ is available. Therefore this larger scale solution can be used to force the local model along $\Gamma$. The formulation of the problem which is solved in that approach is :

$$
\begin{gathered}
L_{e x t} u_{e x t}=f_{\text {ext }} \\
\text { then }
\end{gathered} \begin{array}{ll}
\text { in } \Omega_{e x t} \cup \Omega_{l o c} \times[0, T] \\
\begin{cases}L_{l o c} u_{l o c}=f_{l o c} & \text { in } \Omega_{l o c} \times[0, T] \\
B u_{l o c}=B u_{e x t} & \text { on } \Gamma \times[0, T]\end{cases}
\end{array}
$$

This interaction between the two models can be performed on-line (the two models are run together) or off-line (the external solution is taken from an archive). In the case of an on-line interaction, $u_{\text {ext }}$ is available at every external model timestep, while it is generally subsampled or averaged (i.e. of lesser quality) in the case of an off-line interaction, in order to limit the storage volume.

Note that this problem (7) is a particular case of the open boundary problem (6). It is different from the target problem (5) because $u_{\text {ext }}$ is computed not only on $\Omega_{e x t}$ but on the global domain $\Omega_{e x t} \cup \Omega_{l o c}$. Therefore both the external and the local equations are supposed to be relevant in $\Omega_{l o c}$. This assumption can be rather reasonable in the particular case where both models are identical except for the resolution, 
and $u_{e x t}$ can be in that case a correct approximation of $u_{l o c}$. However, as mentioned previously, $L_{e x t}$ and $L_{l o c}$ generally differ, as well as the forcing terms and the bathymetries. The quality of $u_{e x t}$ is then lesser, which will degrade the estimation of $u_{l o c}$. Moreover, since this approach is only one-way, $u_{l o c}$ never acts on $u_{e x t}$, and the external model cannot be improved.

Usual two-way nesting. An immediate possibility to address this shortcoming is to add a feedback from the local model onto the external one. Formulation (7) then becomes

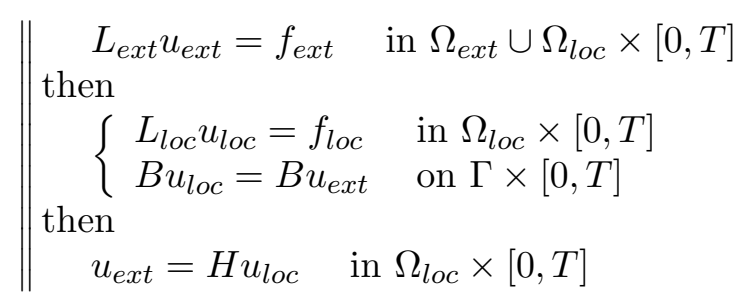

where $H$ is an update operator, mapping $u_{l o c}$ from its time and space grid onto the grid of the external model. This implies of course that the external model is fully available, and that both models are run together with on-line interaction. The update can be performed at each external model timestep, or less frequently.

In this approach, the local solution has some influence onto the external one, the goal being to get closer to the target problem (5) without having to modify the external model.

Full coupling. As mentioned previously, the correct approach should be to solve (5). However this implies first to modify the external model by defining an open boundary on $\Gamma$ in order to avoid overlapping $\Omega_{l o c}$, and also to find an interaction procedure that makes $u_{l o c}$ and $u_{e x t}$ satisfy the regularity conditions on $\Gamma$. We will see in $\S 4.2$ how this can be done. Such methods are quite recent, and are not yet disseminated in the ocean and atmosphere modelling community.

\subsection{A numerical example}

Let us now illustrate the different preceding approaches in the very simple case of a 1-D ordinary differential equation. The problem is :

$$
\left\{\begin{array}{l}
\left.-\nu(x) u^{\prime \prime}(x)+u(x)=\sin n \pi x \quad x \in\right] 0,1[ \\
u(0)=u(1)=0
\end{array}\right.
$$

The local domain we are interested in is $\left.\Omega_{l o c}=\right] a, b\left[\right.$; hence $\Omega_{e x t}=$ ] $0, a[\cup] b, 1\left[. \nu(x)\right.$ is displayed on Figure 2a. It is equal to $\nu_{0}$ in $\Omega_{e x t}$, and 
$\nu_{0} / \sqrt{2}$ in $\Omega_{l o c}$, except in two small transition zones of width $\delta$, where it varies smoothly between $\nu_{0}$ and $\nu_{0} / \sqrt{2}$. This problem has a unique solution (Brezis, 1983), denoted $u_{r e f}$, which is plotted on Figure 2b. The elliptic nature of this problem amplifies the influence of the boundary conditions, which will help highlighting the differences between the nesting approaches described in $\S 2$.

Open boundary problem. $\quad$ Solve $-\nu(x) u_{o b c}^{\prime \prime}(x)+u_{o b c}(x)=\sin n \pi x$, $x \in] a, b[$, with OBCs at $a$ and $b$. Such OBCs can be for example Dirichlet conditions $u_{o b c}(a)=\alpha_{0}, u_{o b c}(b)=\beta_{0}$, or Neumann conditions $u_{o b c}^{\prime}(a)=\alpha_{1}, u_{o b c}^{\prime}(b)=\beta_{1}$. If the external data are perfect $\left(\left[\alpha_{0}, \beta_{0}\right]=\right.$ $\left[u_{r e f}(a), u_{r e f}(b)\right]$ or $\left.\left[\alpha_{1}, \beta_{1}\right]=\left[u_{r e f}^{\prime}(a), u_{r e f}^{\prime}(b)\right]\right)$ then we get the true solution $u_{r e f}$. We have plotted in Figure $2 \mathrm{~b}$ the case of imperfect Dirichlet data $\alpha_{0}=\beta_{0}=0$.

One-way / two-way nesting. Since the problem is not time dependent, both one-way and two-way approaches yield the same solution $u_{n e s}$, defined by :

$$
\begin{aligned}
& \left\{\begin{array}{l}
\left.-\nu_{0} u_{\text {ext }}^{\prime \prime}(x)+u_{\text {ext }}(x)=\sin n \pi x, \quad x \in\right] 0,1[ \\
u_{\text {ext }}(0)=u_{\text {ext }}(1)=0
\end{array}\right. \\
& \left\{\begin{array}{l}
\left.-\nu(x) u_{\text {nes }}^{\prime \prime}(x)+u_{\text {nes }}(x)=\sin n \pi x, \quad x \in\right] a, b[ \\
B_{a} u_{\text {nes }}(a)=B_{a} u_{\text {ext }}(a) \text { and } B_{b} u_{n e s}(b)=B_{b} u_{e x t}(b)
\end{array}\right.
\end{aligned}
$$

We have plotted in Figure $2 \mathrm{~b}$ the cases $B_{a}=B_{b}=I d$ and $B_{a}=B_{b}=$ $\partial / \partial n$. As can be seen clearly, these methods, which are all supposed to approximate the true problem (9), yield quite different solutions, which can differ from $u_{\text {ref }}$ both in $\Omega_{l o c}$ and $\Omega_{e x t}$. Note also that the true problem (9), reformulated as (5), requires two BCs at $a$ and $b$, while the approximate formulations require only one $\mathrm{BC}$.

The same type of comparison is displayed in Figure 3, but for the realistic testcase of a high resolution model of the bay of Biscay coupled with an eddy-permitting model of the North Atlantic.

\section{The open boundary problem}

Let us now focus on the main point, central in all approaches, namely the choice of the open boundary operators $B$ in (6)-(7)-(8). This is a difficult problem, which has been the subject of numerous studies for more than 30 years, ranging from purely mathematical approaches to specific modelling applications. Mathematical results are often obtained for simplified equations (e.g. linearized and/or inviscid). They generally address the derivation of OBCs, and the well-posedness of the model equations using these OBCs. Note that the well-posedness of the system 

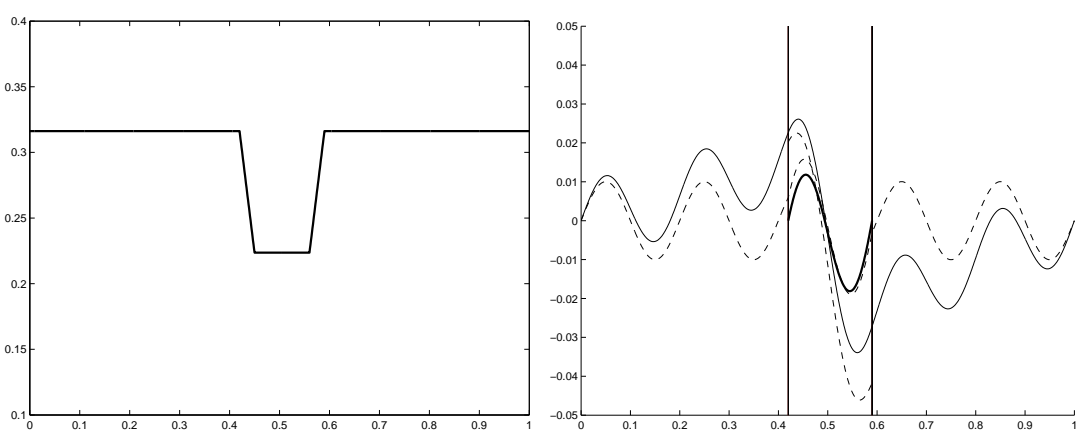

Figure 2. a) $\nu(x)$ for eq.(9); b) The different solutions (see text) : $u_{\text {ref }}$ (solid line), $u_{o b c}$ (thick solid line), $u_{n e s}$ with Dirichlet and Neumann OBCs (dashed lines). The two vertical lines correspond to $x=a$ and $x=b$.

Ete

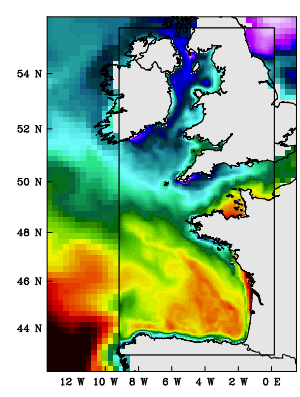

Ete

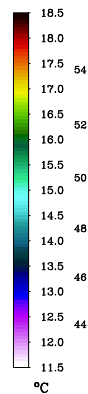

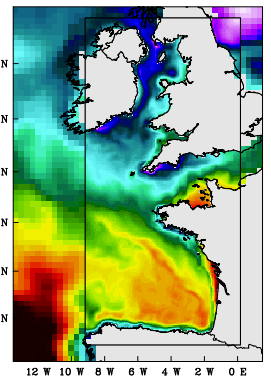

Ete

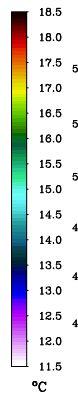

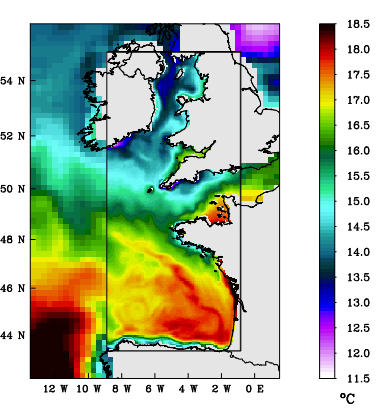

Figure 3. Averaged temperature at $z=30 \mathrm{~m}$ in spring 1998 , in a $1 / 15^{\circ}$ regional model of the bay of Biscay interacting with a $1 / 3^{\circ}$ model of the north Atlantic. The internal rectangle corresponds to the limits of the regional model. The model of the north Atlantic is only partially shown. Three interactions procedures are compared (from S. Cailleau, 2004).

ensures the uniqueness of the solution and its stability with regard to initial datum, but does not give any information on its accuracy nor relevance with regard to the "true" solution $u_{r e f}$. On the other hand, numerical studies can use complex realistic models, but their results seem often dependent on the test cases. We present here a brief overview of usual OBCs, and give a tentative explanation of their performance through the point of view of hyperbolic systems. The contents of this section is discussed in much more details in Blayo and Debreu (2005). 


\subsection{Usual OBCs}

Several reviews of OBCs are available, either for ocean and atmosphere models or in a more general context. Let us mention for instance the introductory parts of the papers by Palma and Matano (1998), Marchesiello et al. (2001), Treguier et al. (2001), or the review papers by Givoli (1991), Tsynkhov (1998) or Holdstad and Lie (1999). OBCs are often classified roughly into two categories : global OBCs are usually accurate, but computationally expensive and difficult to implement; local OBCs are much cheaper and easier to implement, but also generally much less accurate and mathematically justified. We will give now briefly a list of OBCs used in the context of ocean and atmosphere modelling.

Relaxation methods. The goal of this widely used class of OBCs is to relax the model solution $\phi$ towards the external data $\phi^{\text {ext }}$ on (or in the vicinity of $) \Gamma$. The most brutal way to do this is to impose $\phi=\phi^{\text {ext }}$ on $\Gamma$, i.e. to use a Dirichlet (or clamped) boundary condition. Such a condition is often used in particular in the context of one-way nesting. However, a major drawback of this method is that the outflowing information is totally determined by these external data, and does not depend at all on the internal solution. Therefore part of the outgoing information will be reflected into the domain as soon as the external data is not perfectly consistent with the internal dynamics. One of the conclusions of a comparative study by Röed and Cooper (1987) in the context of a simple linear barotropic ocean model is that such a clamped BC should be avoided in most applications.

It is frequent in practical applications to use a more progressive method, called flow relaxation scheme. This approach consists in extending the computational domain $\Omega_{\text {loc }}$ by defining an additional domain $\Omega_{s}$ (the sponge layer), which interface with $\Omega_{\mathrm{loc}}$ is $\Gamma$. In the original method proposed by Davies (1976), the model equations are numerically solved on $\Omega_{\text {loc }} \cup \Omega_{s}$, and the solution in $\Omega_{s}$ is replaced at each timestep by

$$
(1-\alpha) \phi+\alpha \phi^{\text {ext }}
$$

where $\alpha$ is a relaxation function increasing from 0 on $\Gamma$ to 1 far enough from $\Gamma$. While primarily designed for discretized equations, it can be shown easily (e.g. Martinsen and Engedahl, 1987) that this correction scheme can also be interpreted as adding a nudging term to the original model equations

$$
\frac{\partial \phi}{\partial t}+F(\phi)=0
$$


which become

$$
\frac{\partial \phi}{\partial t}+F(\phi)+K\left(\phi-\phi^{\mathrm{ext}}\right)=0
$$

where $K$ is a positive function, null on $\Omega_{\text {loc }}$ and increasing away from $\Gamma$ ( $K$ depends on $\alpha$ and on the time-discretization scheme). Relaxation methods are often performed jointly with a sponge layer approach, which means that the model viscosity is artificially increased in $\Omega_{s}$, in order to damp the local turbulent activity. Relaxation generally appears to be one of the best methods in comparative numerical studies (e.g. Röed and Cooper, 1987; Palma and Matano, 1998; Nycander and Döös, 2003).

Two drawbacks of these methods must however be emphasized. The first one is the increase of the computational cost induced by the additional layers $\Omega_{s}$. The ratio of this additional cost to the cost of the initial model is roughly equal to $\left|\Omega_{s}\right| /\left|\Omega_{\text {loc }}\right|$, and can either be negligible or reach some tens of percents, depending on the configuration. The second drawback is the empirical aspect of the governing equation (13) in the sponge layer.

Finally, note also that perfectly matched layer (PML) methods, which have been proposed quite recently in the context of electromagnetism (Berenger, 1994), can be seen as an improvement of relaxation methods. This methodology consists basically in a convenient splitting of the equations with addition of relaxation terms with well-chosen coefficients. PML approach has been applied to the Euler equations (Hu, 1996, 2001) and to the shallow water equations (Darblade et al., 1997; Navon et al., 2004), and leads to improved results in academic test cases. It must now be validated in realistic configurations to get a better evaluation of its actual effectiveness.

Radiation methods. A very popular class of OBCs are radiation methods. They are based on the Sommerfeld condition :

$$
\frac{\partial \phi}{\partial t}+c \frac{\partial \phi}{\partial n}=0
$$

which corresponds to the transport of $\phi$ through $\Gamma$ ( $n$ is the outward normal vector) with the velocity $c$.

Orlanski (1976) proposed a numerical implementation of this condition for complex flows, including an adaptive evaluation of $c$. A number of variants were then derived, using alternative computations of $c$, and/or taking into account the tangential derivative, and/or including an additional relaxation term (e.g. Camerlengo and O'Brien, 1980; Miller and Thorpe, 1981; Raymond and Kuo, 1984; Barnier et al., 1998; Marchesiello et al., 2001). 
Such radiation methods are frequently used in ocean and atmosphere modelling. However their relevance for such complex flows is far from obvious. Their reputation is split : they have proved to give rather poor results in several comparative studies (e.g. Röed and Cooper, 1987; Palma and Matano, 1998; Nycander and Döös, 2003), while they seem to have some efficiency in others (e.g. Marchesiello et al., 2001; Tréguier et al., 2001). In fact the Sommerfeld condition is justified only in the context of wave equations with a constant phase velocity (Blayo and Debreu, 2005). Applying such a condition to variables which do not satisfy at all such equations results in a fundamental nonlinearity, which has been recently pointed out by Nycander and Döös (2003). Therefore this condition cannot be mathematically justified in the context of ocean and atmosphere modelling. However, its actual implementations give an important role to external data. As indicated previously, the radiation velocity $c$ is evaluated at each timestep and at each gridpoint on the open boundary. If $c$ is inward, the model variable is generally set to the corresponding external value: $\phi=\phi^{\text {ext }}$, or strongly relaxed towards it:

$$
\frac{\partial \phi}{\partial t}=-\frac{\phi-\phi^{\mathrm{ext}}}{\tau_{\mathrm{in}}}
$$

where $\tau_{\text {in }}$ is a short relaxation timescale. If $c$ is outward, then the radiation equation is applied, but often with the addition of a relaxation term :

$$
\frac{\partial \phi}{\partial t}+c \frac{\partial \phi}{\partial n}=-\frac{\phi-\phi^{\text {ext }}}{\tau_{\text {out }}}
$$

where $\tau_{\text {out }}$ is a longer relaxation timescale. In their careful analysis of a simulation of the Atlantic ocean, Tréguier et al. (2001) have observed that $c$ behaves in some sense like a white noise, and is directed inwards about half of the time at any location on the open boundaries. Therefore the model solution at the open boundary never departs significantly from the external data, and the radiation condition acts in fact nearly as a clamped condition. So it is probably the strong influence of the external data through the additional relaxation term in the radiation conditions that gives them most of their practical efficiency, rather than the radiation procedure.

Flather condition. Flather (1976) proposed an OBC for 2-D barotropic flows, which is often classified within the family of radiation conditions. This condition can be obtained by combining the Sommerfeld condition for the surface elevation $\eta$ (with surface gravity waves phase speed)

$$
\frac{\partial \eta}{\partial t}+\sqrt{g h} \frac{\partial \eta}{\partial n}=0
$$


with a one-dimensional approximation of the continuity equation

$$
\frac{\partial \eta}{\partial t}+h \frac{\partial v_{n}}{\partial n}=0
$$

where $g$ is the gravity, $h$ is the local water depth and $v_{n}$ is the normal component of the barotropic velocity. Substracting (17) to (18) and integrating through $\Gamma$, one obtains:

$$
v_{n}-\sqrt{\frac{g}{h}} \eta=v_{n}^{\mathrm{ext}}-\sqrt{\frac{g}{h}} \eta^{\mathrm{ext}}
$$

The Flather condition has been used in several comparative studies (e.g. Palma and Matano, 1998; Marchesiello et al., 2001; Nycander and Döös, 2003), and it always appears to be one of the most efficient conditions.

Model adapted methods. A striking aspect of radiation and relaxation methods is that the OBCs do not depend on the model equations. On the opposite, other methods provide OBCs which are adapted to the system. However, since they are more complicated to handle, the use of such methods is quite rare and restricted to simple 1-D or 2-D models, and has never been extended to our knowledge to realistic primitive equations systems.

This is the case of characteristic waves amplitudes methods (sometimes called Hedström methods), designed for hyperbolic systems. The basic idea consists in choosing for OBCs the original set of model equations with as few approximations as possible. Since the only quantities that cannot be evaluated by the model alone are the incoming characteristics (see §3.2) the approximations must concern only these terms, and eventually the viscous terms if the model is not inviscid. This results in setting to zero (or to a value deduced from external data) the normal derivative of the incoming characteristic variables on $\Gamma$. Several papers developed this idea these last years in the context of direct numerical simulation of compressible Euler and Navier-Stokes equations, with apparently good experimental results (Poinsot and Lele, 1992; Bruneau, 2000; Bruneau and Creusé, 2001). In the context of ocean modelling, it is compared to other OBCs by Röed and Cooper (1987), Jensen (1998) and Palma and Matano (1998), and leads to rather good results.

Another important family of methods are absorbing conditions, which are exact relations satisfied by the outgoing quantities at the open boundary. In a reference paper, Engquist and Majda (1977) give a general method for obtaining such relations, using time and space Fourier transforms. However, these conditions are generally global in time and space, and cannot be used just as it is in practice. That is why they 
must be approximated to give tractable local conditions. A strong interest of this approach is its sound mathematical foundation, and its practical efficiency in several domains of applications. Several papers have recently readdressed the derivation of absorbing BCs for the inviscid shallow water system, and obtain apparently quite good numerical results (Lie, 2001; McDonald, 2002, 2003; Nycander and Döös, 2003).

\subsection{An hyperbolic point of view}

When attempting to draw some synthesis of the numerous previous studies on OBCs, two keypoints stand out, which seem to be necessary constituents for any good OBC. The first point is that good results are obtained when taking primarily into account the hyperbolic part of the dynamics, and therefore when working on incoming characteristic variables. The second point is that this must be associated with a consistent use of some external data.

Incoming characteristic variables. Let us first introduce some standard definitions concerning hyperbolic systems. The general form of such a system is

$$
\frac{\partial \Phi}{\partial t}+A(\Phi) \frac{\partial \Phi}{\partial x}=F
$$

where $\Phi(x, t)$ is a vector of $n$ functions, $A(\Phi)$ is a $n \times n$ matrix of functions of $\Phi$, and $F$ is a forcing term. For the system to be hyperbolic, $A$ must have $n$ real eigenvalues and $n$ distinct eigenvectors. Let $W_{k}$ the $k^{\text {th }}$ left eigenvector of $A$, corresponding to the $k^{\text {th }}$ eigenvalue $\lambda_{k}$ : $W_{k}^{T} A=\lambda_{k} W_{k}^{T}$. Multipliying (20) on the left by $W_{k}^{T}$, one gets:

$$
W_{k}^{T} \frac{d_{k} \Phi}{d t}=W_{k}^{T} F \quad \text { with } \quad \frac{d_{k}}{d t}=\left(\frac{\partial}{\partial t}+\lambda_{k} \frac{\partial}{\partial x}\right)
$$

The operator $d_{k} / d t$ represents a total (or directional) derivative in the direction defined by $\frac{d x}{d t}=\lambda_{k}$. To the hyperbolic system (20) correspond $n$ such families of curves, which are called characteristic curves of the system. If the system (20) is linear with constant coefficients, i.e. if $A$ is a constant matrix, one can define the new variables $w_{k}(x, t)=W_{k}^{T} \Phi(x, t)$. (20) is then equivalent to the system of $n$ uncoupled transport equations:

$$
\frac{\partial w_{k}}{\partial t}+\lambda_{k} \frac{\partial w_{k}}{\partial x}=W_{k}^{T} F \quad k=1, \ldots, n
$$

The characteristic curves in that case are the lines $x-\lambda_{k} t=$ constant, along which the $w_{k}$ (called characteristic variables or Riemann invariants) are conserved. One can notice that, at a given boundary, these 
characteristic variables will be either inflowing or outflowing, depending on the sign of $\lambda_{k}$.

A fundamental point is that, for a hyperbolic open boundary problem to be well-posed, one must prescribe as many boundary conditions as the number of incoming characteristics. This result is in fact quite intuitive : the solution can be decomposed into outgoing and incoming characteristics; information on the former is available within the computation domain, and no additional condition is required, while information on the latter is not available, and mustbe specified.

Consistency with external data. The second keypoint concerns the connection with external data. It appears that a reasonable choice consists in imposing the consistency locally all along the boundary. This means that the OBC is of the form

$$
B \phi=B \phi^{\text {ext }}
$$

where $B$ is the open boundary operator. $B=I d$ corresponds to the continuity of $\phi$ through the boundary, and $B=\partial / \partial n$ to the continuity of the flux. Such a formulation (23) is quite natural for example if we consider that the external data $\phi^{\text {ext }}$ represents some steady state or far field solution $\phi^{\infty}$. In that case, as detailed for example by Engquist and Halpern (1988), if we want the model solution to converge to the steady state solution as $t \rightarrow \infty$, then the OBC must also be satisfied by $\phi^{\infty}$.

Used together with the point of view of characteristic variables presented previously, this condition (23) leads to recommending OBCs of the form

$$
B w=B w^{\text {ext }}
$$

where $w$ is any incoming characteristic variable of the governing equations.

The extension to non-hyperbolic systems, like for example the NavierStokes equations, is not trivial. A logical approximation consists however in considering only the hyperbolic part of thesystem, and to use the same procedures as for the hyperbolic case.

Revisiting usual OBCs. The preceding criteria give a new light on usual OBCs. It appears indeed that :

- the Sommerfeld condition (14) corresponds to prescribing to zero the incoming characteristic of the wave equation. That is why it is legitimate for wave equations but not for other systems. 
- the Flather condition (14) corresponds to specifying the value of the incoming characteristic of the shallow water system, fulfilling the criterion (24) with $B=I d$ the identity operator.

- absorbing conditions are closely linked to incoming characteristic variables, and the conditions proposed by McDonald $(2002,2003)$ and Nycander and Döös (2003) can be written under the form (24).

- characteristic waves amplitudes methods do also meet the preceding point of view.

- since relaxation methods are not local conditions, the criterion (24) does not apply directly. However, it is obvious from (11) that the transition from $\phi$ to $\phi^{\text {ext }}$ is smooth as soon as the additional domain $\Omega_{s}$ is large enough. Similarly the problem of specifying incoming characteristics and evacuating outcoming characteristics at the open boundary is treated implicitely : the values of the incoming characteristics are computed within $\Omega_{s}$, using the relaxed solution, while the outgoing characteristics are not directly affected when reaching $\Gamma$ but are relaxed in $\Omega_{s}$ towards their corresponding external values, and damped by the increased dissipation.

Details on these aspects, as well as an application of the criterion (24) to shallow-water and primitive equations systems, are discussed in Blayo and Debreu (2005).

\subsection{Some practical remarks}

- It is important to note that we discussed here only the continuous form of the equations. However discretized models contain spurious numerical modes, which nature is different from that of physical modes, and which have to be handled by the OBCs. Therefore, once the continuous form of the OBCs is chosen, one has to perform some specific work in order to adapt their numerical implementation to the numerical schemes of the model. This difficulty is probably also a reason for the efficiency of relaxation and radiation-relaxation methods, which tend to automatically damp these non-physical modes.

- Incoming information is entirely given by the external solution $\phi^{\text {ext }}$. Therefore the quality of these data is of course an important point in the performance of a regional modelling system.

- Another important practical aspect in a regional modelling system is the initialization problem. The initial condition is generally built 
by interpolation of a larger scale solution, which is not perfectly consistent with the local model. This can yield an adjustment phase which can be quite long, and which pollutes the model solution. A way to avoid (or limit) this problem is to add some relevant constraints in the computation of the initial condition, as done for instance by Auclair et al. (2000) using an inverse approach. This aspect is presently the subject of numerous studies.

\section{Two-way interaction}

\subsection{Two-way nesting}

As explained in $\S 2$, the usual two-way method differs from the preceding one-way method by the addition of an update procedure. This supplementary step aims at improving $u_{\text {ext }}$ by modifying it locally using $u_{\text {loc }}$. This retroaction from the local model onto the external model is performed every external model timestep, or less frequently. The update operator generally replaces the values of $u_{\text {ext }}$ at gridpoints located in $\Omega_{\text {loc }}$ by copying the corresponding values of $u_{\text {loc }}$, eventually after some time and space averaging. Such an update is quite brutal, and in particular does not ensure the balance of mass and tracers fluxes through $\Gamma$. For example, $\int_{\Gamma} \mathbf{U}_{\text {loc }} \cdot \mathbf{n} \neq \int_{\Gamma} \mathbf{U}_{\text {ext }} . \mathbf{n}$, where $\mathbf{U}$ denotes the velocity. That is why a flux correction step is often added, which generally modifies $u_{\text {loc }}$ to distribute the flux misfit all along $\Gamma$, to get finally a local solution $u_{\mathrm{loc}}^{*}$ which is in flux balance with $u_{\text {ext }}$.

The two-way method generally decreases the difficulties that can be encountered by the one-way method (in particular the instabilities along $\Gamma$ ), and seems to improve the model solution. That is why it is recommended to use it as far as possible rather than one-way interaction. However, it is clear that the solution provided by this usual two-way nesting is not solution of the original problem (5): before the flux correction step, the connection between $u_{\text {ext }}$ and $u_{\text {loc }}$ is not differentiable, because their fluxes are not balanced; after the flux correction step, the connection is no more continuous because $u_{\text {loc }}$ has been modified into $u_{\text {loc }}^{*}$, which in addition does not satisfy any longer the local model equations (1).

\subsection{Full coupling - Schwarz methods}

Obtaining a solution of the original problem (5) is much more difficult and expensive than what is done in the above usual algorithms. This is mainly due to the fact that, since the local and external model equations are different, their domains of application should not overlap. Therefore 
the external model, which is generally available in a configuration fully overlapping $\Omega_{\text {loc }}$, must be modified to add an open boundary. Moreover, once this is done, one has to find and implement an algorithm ensuring that the solutions $u_{\text {ext }}$ and $u_{\text {loc }}$ will satisfy the desired regularity conditions through $\Gamma$.

These difficulties explain that this problem has never been addressed before in ocean and atmosphere modelling. This can be done however within the mathematical framework of domain decomposition methods. These methods have been intensively studied and developed since the end of the eighties due to the advent of parallel computers. Without going into details, let us present the global-in-time non-overlapping Schwarz algorithm, which seems well suited for our ocean coupling problem. This iterative algorithm can be written as follows :

$$
\begin{aligned}
\left\{\begin{array}{rll}
L_{l o c} u_{l o c}^{n+1} & =f_{l o c} & \text { in } \Omega_{l o c} \times[0, T] \\
u_{l o c}^{n+1} & \text { given } & \text { at } t=0 \\
B_{l o c} u_{l o c}^{n+1} & =B_{l o c} u_{\text {ext }}^{n} & \text { on } \Gamma \times[0, T]
\end{array}\right. \\
\text { and }\left\{\begin{array}{rll}
L_{e x t} u_{\text {ext }}^{n+1}=f_{\text {ext }} & \text { in } \Omega_{\text {ext }} \times[0, T] \\
u_{\text {ext }}^{n+1} & \text { given } & \text { at } t=0 \\
B_{\text {ext }} u_{\text {ext }}^{n+1} & =B_{\text {ext }} u_{l o c}^{n} & \text { on } \Gamma \times[0, T]
\end{array}\right.
\end{aligned}
$$

where the superscripts denote the number of iterations, and $B_{l o c}$ and $B_{\text {ext }}$ are interface operators to be chosen. Note that, at each iteration, the two models can be run in parallel over the whole time window $[0, T]$. If no parallel computer is available, the interface condition for $u_{\text {ext }}$ can be replaced for example by $B_{\text {ext }} u_{e x t}^{n+1}=B_{e x t} u_{l o c}^{n+1}$, which prevents parallelism but increases the convergence rate of the algorithm.

This rate closely depends of the choice of $B_{l o c}$ and $B_{e x t}$. An obvious possibility is to choose the operators $I d$ and $\partial / \partial n$. Therefore, once the algorithm has converged, its solution will satisfy (5). However the convergence can be quite slow and, given the computational burden of ocean models, one probably cannot afford numerous iterations of such an algorithm. That is why the choice of the interface operators must be optimized. A simple but quite efficient possibility is to use Robin conditions : $B_{l o c}=\partial / \partial n+r_{l o c} I d$ and $B_{e x t}=\partial / \partial n+r_{e x t} I d$ with $r_{l o c} \neq r_{e x t}$. This ensures the desired regularity as previously for the converged solution, but a good choice of the coefficients $r_{l o c}$ and $r_{e x t}$ can greatly speed up the convergence. More sophisticated approaches can be used to determine good interface operators, which are closely linked to characteristic methods and absorbing conditions. Martin (2003) applied such approaches to 2-D tracer equations and to the shallow-water system. She derived very efficient operators, which ensure the convergence of the 
algorithm in some very few iterations. Development of such algorithms for realistic ocean models is ongoing research work.

\section{Software tools}

Designing nested or coupled systems starting from existing models is quite a difficult and time-consuming practical task. However several software tools have been developed these last years, which automatically manage an important part of the job.

The AGRIF package ${ }^{1}$ (Debreu et al., 2004a) allows an easy integration of mesh refinement capabilities within any existing finite-difference model written in Fortran. One can therefore design one-way and twoway multiply-nested systems, with the possibility of adaptive regridding, without reprogramming the model. This package is presently implemented into several operational ocean models.

General couplers can also be used to implement nested systems, especially in the case when the local and external model codes are totally different. The user has then to prescribe the structure of the coupling algorithm and the interactions between the different objects, but at a rather high level, without having to go too much into programming details. In the context of geophysical fluids, we can cite for instance PALM $^{2}$ or $\mathbf{M p C C I}^{3}$.

\section{Data assimilation and nesting}

Along with the development of nested ocean modelling systems, the problem of assimilating data within these systems is presently strongly emerging. Addressing this difficult problem is out of the scope of this note. Let us however point out a few related issues.

- The exact mathematical formulation of the data assimilation problem for one-way or two-way nested systems is far from obvious. A first attempt in this direction for the 4D-Var approach can be found in Debreu et al. (2004b). Concerning the stochastic approach, interesting ideas can probably be found in the theories of multiresolution stochastic models and multiscale estimation.

- Several ad-hoc procedures are already in use in numerous systems. A possibility is to perfom the assimilation only on one grid (the largest or the finest) of the system. Another way is to "hide" the

\footnotetext{
${ }^{1}$ http://www-lmc .imag.fr/IDOPT/AGRIF

${ }^{2}$ http://www.cerfacs.fr/ palm

${ }^{3}$ http://www.mpcci.org
} 
grid interaction process and to make the assimilation globally on a multiresolution state vector (e.g. Barth et al., 2004).

- It is possible in a variational method to manage simultaneously the coupling problem and the assimilation problem. See for instance Bounaim (1999) or Taillandier et al.(2004).

- In a multiresolution modelling system, one has to choose which data are assimilated on which grid. Since the model dynamics depends on the grid resolution, and since the data themselves have often been collected or processed with some spatial and temporal resolution, this choice is not obvious and has consequences on the quality of the identified solution.

\section{References}

Auclair, F., S. Casitas, and P. Marsaleix, 2000: Application of an inverse method to coastal modelling. J. Atmos. Oceanic Technol., 17, 1368-1391.

Barnier, B., P. Marchesiello, A.P. de Miranda, J.M. Molines, and M. Coulibaly, 1998: A sigma coordinate primitive equation model for studying the circulation in the South Atlantic I, Model configuration with error estimates. Deep Sea Res., 45, $543-572$.

Barth, A., A. Alvera-Azcarate, J.-M. Beckers, M. Rixen, L. Vandenbulke and Z. Ben Bouallegue, 2004: Multigrid state vector for data assimilation in a two-way nested model of the Ligurian sea. 36th International Liege Colloquium on Ocean Dynamics.

Bennett, A.F., 2002: Inverse modeling of the ocean and atmosphere. Cambridge University Press, 2002.

Berenger, J.-P., 1994: A perfectly matched layer for the absorption of electromagnetic waves. J. Comput. Phys., 114, 185-200.

Blayo, E., and L. Debreu, 2005: Revisiting open boundary conditions from the point of view of characteristic variables. Ocean Modelling, 9, 231-252.

Bounaim, A., 1999: Méthodes de décomposition de domaine: application à la résolution de problèmes de contrôle optimal. PhD thesis, Université Grenoble 1.

Brezis, H., 1983: Analyse fonctionnelle. Masson.

Bruneau, C.-H., 2000: Boundary conditions on artificial frontiers for incompressible and compressible Navier-Stokes equations. Math. Mod. and Num. Anal., 34, 303314.

Bruneau, C.H., and E. Creusé, 2001: Towards a transparent boundary condition for compressible Navier-Stokes equations. Int. J. Numer. Meth. Fluids, 36, 807-840.

Cailleau, S., 2004: Validation de méthodes de contrainte aux frontières d'un modèle océanique : application à un modèle hauturier de l'Atlantique Nord et à un modèle régional du Golfe de Gascogne. PhD thesis, Université Grenoble 1.

Camerlengo, A.L., and J.J. O'Brien, 1980: Open boundary conditions in rotating fluids. J. Comp. Phys., 35, 12-35.

Darblade, G., R. Baraille, A.-Y. Le Roux, X. Carton, and D. Pinchon, 1997: Conditions limites non réfléchissantes pour un modèle de Saint-Venant bidimensionnel barotrope linéarisé. C.R. Acad. Sci. Paris, Série 1, 324, 485-490. 
Davies, H.C., 1976: A lateral boundary formulation for multi-level prediction models. Quart. J. R. Meteorol. Soc., 102, 405-418.

Debreu, L., C. Vouland and E. Blayo, 2004a: AGRIF: Adaptive Grid Refinement in Fortran. To appear in Computers and Geosciences.

Debreu, L., Y. De Visme and E. Blayo, 2004b: 4D Variational data assimilation for locally nested models. In preparation.

Engquist, B., and A. Majda, 1977: Absorbing boundary conditions for the numerical simulation of waves. Math. Comp., 31, 629-651.

Engquist, B., and L. Halpern, 1988: Far field boundary conditions for computation overlong time. Appl. Num. Math., 4, 21-45.

Flather, R.A., 1976: A tidal model of the north-west European continental shelf. Mem. Soc. R. Sci. Liège, 6(10), 141-164.

Givoli, D., 1991: Non-reflecting boundary conditions. J. Comp. Phys., 94, 1-29.

Hedström, G.W., 1979: Nonreflecting boundary conditions for nonlinear hyperbolic system. J. Comp. Phys., 30, 222-237.

Holstad, A., and I. Lie, 1999: On transparent boundary conditions and nesting for ocean models. Research report 91, Norwegian Meteorological Institute, Oslo, Norway.

Hu, F. Q., 1996: On absorbing boundary conditions for linearized Euler equations by a perfectly matched layer. J. Comp. Phys., 129, 201-219.

Hu, F. Q., 2001: A stable perfectly matched layer for linearized Euler equations in unsplit physical variables. J. Comp. Phys., 173, 455-480.

Jensen, T., 1998: Open boundary conditions in stratified ocean models. J. Mar. Sys., 16, 297-322.

Lie, I., 2001: Well-posed transparent boundary conditions for theshallow water equations. App. Num. Math., 38, 445-474.

Marchesiello, P., J. McWilliams, and A. Shchepetkin, 2001: Open boundary conditions for long-term integration of regional oceanic models. Ocean Modelling, 3, 1-20.

Martin, V., 2003: Méthodes de décomposition de domaine de type relaxation d'ondes pour des équations de l'océanographie. PhD thesis, Université Paris 13.

Martinsen, E.A., and H.E. Engedahl, 1987: Implementation and testing of a lateral boundary scheme as an open boundary condition in a barotropic ocean model. Coastal Eng., 11, 603-627.

McDonald, A., 2002: A step toward transparent boundary conditions for meteorological models. Mon. Weath. Rev., 130, 140-151.

McDonald, A., 2003: Transparent boundary conditions for the shallow water equations: testing in a nested environment. Mon. Weath. Rev., 131, 698-705.

Miller, M.J., and A.J. Thorpe, 1981: Radiation conditions for the lateral boundaries of limited-area numerical models. Quart. J. R. Meteorol. Soc., 107, 615-628.

Navon, I.M., B. Neta, and M.Y. Hussaini, 2004: A perfectly matched layer approach to the linearized shallow water equations models: the split equation approach. Mon. Weather Rev., 132, 1369-1378.

Nycander, J., and K. Döös, 2003: Open boundary conditions for barotropic waves. $J$. Geophys. Res., 108(C5), 3168-3187.

Orlanski, I., 1976: A simple boundary condition for unbounded hyperbolic flows. $J$. Comp. Phys., 21, 251-269.

Palma, E.D., and R.P. Matano, 1998: On the implementation of passive open boundary conditions for a general circulation model: the barotropic mode. J. Geophys. Res., 103(C1), 1319-1341. 
Poinsot, T., and S.K. Lele, 1992: Boundary conditions for subsonic Navier-Stokes calculations. J. Comp. Phys., 101, 104-129.

Raymond, W.H., and H.L. Kuo, 1984: A radiation boundary condition for multidimensional flows. Quart. J. R. Met. Soc., 110, 535-551.

Röed, L.P., and C. Cooper, 1987: A study of various open boundary conditions for wind-forced barotropic numerical ocean models, in Three-dimensional models of marine andestuarine dynamics, edited by J.C.J. Nihoul and B.N. Jamart, pp. 305335, Elsevier.

Taillandier, V., V. Echevin, L. Mortier and J.-L. Devenon, 2004: Controlling boundary conditions with a four-dimensional variational data assimilation method in a nonstratified open coastal model. Ocean Dyn., 54, 284-298.

Tréguier, A.-M., B. Barnier, A.P. de Miranda, J.-M. Molines, N. Grima, M. Imbard, G.Madec, and C. Messager, 2001: An eddy permitting model of the Atlantic circulation: evaluating openboundary conditions. J. Geophys. Res, 106(C10), 2211522130.

Tsynkhov, S.V., 1998: Numerical solutions of problems on unbounded domains. A review. Appl. Numer. Math., 27, 456-532. 